\title{
Superior immunogenicity of HCV envelope glycoproteins when adjuvanted with cyclic-di-AMP, a STING activator or archaeosomes
}

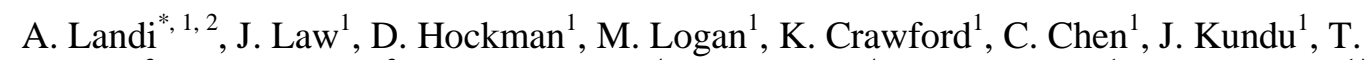
Ebensen $^{3}$, C.A. Guzman ${ }^{3}$, L. Deschatelets ${ }^{4}$, L. Krishnan ${ }^{4}$, D.L.J. Tyrrell ${ }^{1}$, M. Houghton ${ }^{1 *}$

${ }^{1}$ Li Ka Shing Institute of Virology, Department of Medical Microbiology and Immunology, University of Alberta, Edmonton, $A B, T 6 G$ 2E1, Canada ${ }^{2}$ Department of Virology and Department of Immunology, School of Medicine, Ahvaz Jundishapur University of Medical Sciences, Ahvaz, Iran; ${ }^{3}$ Department of Vaccinology and Applied Microbiology, Helmholtz Centre for Infection Research, Braunschweig, Germany; ${ }^{4}$ Immunobiology Department, Human Health Therapeutics, National Research Council Canada, Montreal, Ottawa, ON KIA OR6 Canada

\section{* Corresponding authors:}

Abdolamir Landi MD, PhD and Michael Houghton PhD

Li Ka Shing Institute of Virology, Department of Medical Microbiology \& Immunology, University of Alberta, 6-010 Katz Group-Rexall Centre for Health Research, Edmonton, AB, T6G 2E1, Canada.

Phone: (780) 248-1888

Fax: (780) 492-7521

Email: landi@ualberta.ca and Michael.houghton@ualberta.ca 


\section{Abstract}

2 Three decades after the discovery, hepatitis $\mathrm{C}$ virus (HCV) is still the leading

3 cause of liver transplantation and poses a major threat to global health. In spite of recent

4 advances in the development of direct acting antivirals, there is still a need for a

5 prophylactic vaccine to limit the virus spread and protect at-risk populations, especially

6 in developing countries, where the cost of the new treatments may severely limit access.

7 The use of recombinant HCV glycoproteins E1E2 (rE1E2) in combination with the

8 MF59, an oil-in-water emulsion-based adjuvant, has previously been shown to reduce the

9 rate of chronicity in chimpanzees and to induce production of cross-neutralizing

10 antibodies and cellular immune responses in human volunteers. To further improve

11 neutralizing antibody responses in recipients along with robust $\mathrm{T}$ cell responses, we have

12 explored the immunogenicity of different adjuvants when formulated with the HCV

13 rE1E2 vaccine in mice.

14 Our data show that cyclic di-adenosine monophosphate (c-di-AMP) and

15 archaeosomes elicit strong neutralizing antibodies similar to those elicited using

16 aluminum hydroxide/monophosphoryl lipid A (Alum/monophos. /MPLA) and MF59.

17 However, both c-di-AMP and archaeosomes induced a more robust cellular immune

18 response, which was confirmed by the detection of vaccine-specific poly-functional

$19 \mathrm{CD}^{+} \mathrm{T}$ cells. We conclude that these adjuvants may substantially boost the

20 immunogenicity of our E1E2 vaccine. In addition, our data also indicates that use of a

21 partial or exclusive intranasal immunization regimen may also be feasible using c-di-

22 AMP as adjuvant.

\section{Introduction}

25 Chronic hepatitis $\mathrm{C}$ is the common consequence of infection with the hepatitis $\mathrm{C}$

26 virus (HCV). It is the leading cause for liver transplant and still a major threat to global

27 health [1]. Despite recent progress in direct antiviral drugs (DAA) that significantly

28 improved the cure rate to higher than 90\% [2], the high cost of DAA, especially in

29 developing countries is a major obstacle to control the disease or to stop the spread of the

30 infection among humans [3]. In addition, the potential of occurrence of resistant strains of

$31 \mathrm{HCV}$ after use of the DAAs [4], reinfection of individuals that cleared the virus after 
1 therapy, and the existence of a major population of undiagnosed silent carriers [5, 6] all

2 make the eradication of the disease by the use of DAA alone very unlikely. Indeed, no

3 infectious disease has yet been eradicated or efficiently controlled through the use of

4 therapeutic drugs alone without the introduction of an effective vaccine. Thus,

5 vaccination may be the only solution for controlling the spread of $\mathrm{HCV}$ as has been

6 previously shown for other human-specific pathogens [7].

7 Recently, the use of recombinant antigens-, subunit- or even peptide-based

8 vaccines are becoming more favorable than killed or attenuated viruses as they could be

9 tolerated better and designed more specifically. However, as these antigens are less

10 immunogenic, the presence of adjuvants in the formulation turns out to be more crucial

11 for optimal and adequate immune responses, while also helping to reduce the amount of

12 antigen required. Adjuvants can serve either as a delivery system or as an immune

13 stimulator or both and can skew the immune response toward a Th-2 type humoral

14 response such as aluminum hydroxide (Alum) or a Th-0 type response as in MF59 or a

15 Th-1 type cellular response such as with monophosphoryl lipid A (MPLA).

16 Our previous efforts on the development of a vaccine for HCV have shown that

17 the use of recombinant HCV glycoproteins E1E2 (rE1E2) in combination with MF59-

18 type adjuvants, oil-in-water based emulsions, reduces the rate of chronicity in

19 chimpanzees and induces production of neutralizing antibodies and cellular immune

20 responses in humans [8-10]. In addition, we have also shown that the neutralizing

21 antibodies elicited by rE1E2/MF59 in humans are capable of cross-neutralizing many

22 different clades of $\mathrm{HCV}$ in vitro, thus validating the use of rE1E2 as an optimum

23 immunogenic antigen [11]. To enhance these antibodies in recipients along with a robust

24 T cell response, we have explored the efficacy of other adjuvants in combination with

25 HCV rE1E2. Various adjuvants operating via different pathways such as Alum/MPLA,

26 MF59, cyclic di-adenosine monophosphate (c-di-AMP), and archaeosomes were

27 formulated to immunize mice and the immunogenicity was measured and compared

28 among groups using neutralizing antibody assays and intracellular cytokine production in

29 T cell assays.

30 Alum adjuvants are the oldest and most commonly used adjuvants in humans and

31 have been used with great success with very well established safety and efficacy profiles. 
1 Alum is primarily effective in promoting humoral immunity as a result of its depot

2 effects. Moreover, Alum increases the antigen uptake and stability and induces pro-

3 inflammatory reactions [12-15]. However, Alum is a poor inducer of Th-1 type immunity

4 and for that reason may be supplemented with other adjuvants such as MPLA.

$5 \quad$ MPLA is a derivative of lipid A from bacterial lipopolysaccharide (LPS) and a

6 well-known agonist for toll-like receptor 4 (TLR4). MPLA could skew the immunity

7 toward a Th1-type in combination with other adjuvants [16] and facilitate optimal

8 humoral and cellular responses when it is used in combination with Alum [17]. Another

9 advantage of using MPLA is that it is now approved for use in combination with Alum in

10 a vaccine for human papilloma virus [18].

11 MF59 is a licensed adjuvant. It is an oil-in-water emulsion-based adjuvant that is

12 safe and potent in generating antibodies. MF59-adjuvanted vaccine has recently been

13 used to control a 2009 pandemic of H1N1 influenza. It has been shown that MF59

14 promotes a $\mathrm{CD} 4^{+} \mathrm{T}$ cell response that leads to production of protective antibodies against

15 the H5N1 Influenza strain [19]. In addition, MF59 induces the recruitment of Th-2 type

16 antigen-presenting cells (APCs), increases the antigen uptake by dendritic cells, and

17 activates multiple inflammatory pathways resulting in production of inflammatory

18 cytokines [20, 21].

19 Stimulator of interferon genes (STING) is a signalling molecule that is essential

20 for the production of pro-inflammatory cytokines including type 1 interferon (IFNs). It

21 has been demonstrated that STING can be strongly activated by cyclic dinucleotide

22 molecules and acts as a direct sensor for them when produced by bacteria [22, 23]. Cyclic

23 dinucleotide adenosine monophosphate (c-di-AMP) is a member of the cyclic

24 dinucleotides and acts as a pathogen-associated molecular pattern (PAMP) to induce

25 immunity when it is used in vaccine formulations [24]. In other studies, c-di-AMP was

26 used as a mucosal adjuvant, where it primarily induced dendritic cells and shown to be

27 capable of promoting both humoral and cellular immune responses [25, 26]. This

28 encompasses the stimulation of Th1, Th2 and Th17 cells, as well as the induction of

29 cytotoxic T lymphocytes.

30 Archaea are the third domain of life, distinct from eubacteria, and possess unique

31 highly stable membrane lipids. Different archaeal species optimally grow in harsh 
1 conditions such as high temperature, extreme $\mathrm{pH}$, high-salt water, and anaerobic habitats;

2 they are also stable in the presence of lipases and bile salts [27-29]. Liposomes are closed

3 vesicles that have been used as delivery vehicles for drug and/or antigen cargo. The term

4 archaeosomes was ascribed to liposome vesicles comprised of ether lipids derived from

5 Archaea. Archaeosomes constitute stable antigen delivery vesicles for vaccines. A key

6 feature of archaeosomes is their dual ability to target antigen for processing on the MHC

7 class I pathway, while concomitantly activating APCs costimulation and cytokine

8 production [30]. Entrapment of antigen in archaeosomes induces both robust antibody

9 and cell-mediated immunity through induction of $\mathrm{CD} 4^{+}$and $\mathrm{CD} 8^{+} \mathrm{T}$ cells $[27,31]$.

10 Here we compare the strength and type of immunity that is elicited by a HCV

11 candidate vaccine when associated with different adjuvants in mice.

\section{Materials and Methods}

14 Antigen and vaccine preparation: HCV H77 rE1E2 heterodimer was prepared 15 and purified to $>90 \%$ purity as described before [32]. Then, various adjuvants including 16 Alum/MPLA, MF59, c-di-AMP, and archaeosomes were formulated with H77 rE1E2 to

17 immunize mice. In general, $20 \mu \mathrm{l}$ of adjuvant was added to $20 \mu \mathrm{l}$ of antigen solution

18 (containing $1 \mu \mathrm{g}$ of rE1E2 in PBS) and mixed well before injecting the animal. The

19 amount of each adjuvant was $100 \mu \mathrm{g}$ of Alum $+10 \mu \mathrm{g}$ of MPLA, $10 \mu \mathrm{g}$ c-di-AMP or 100

$20 \mu \mathrm{g}$ archaeosomes. Archaeosomes containing E1E2 protein were prepared by the method

21 of detergent removal using the hydrophobic polystyrene Bio-beads SM adsorbent [33, 34]

22 Where needed, the total volumes of adjuvants were adjusted to $20 \mu \mathrm{l}$ per dose by adding

23 PBS. In case of MF59, $20 \mu 1$ of in-house prepared adjuvant was used.

Immunization: Female CB6-F1 mice (8 mice per group, 6-8 week-old, Charles

25 River laboratories, MA, USA) were immunized with 1-2 $\mu \mathrm{g}$ of antigen mixed in a 1:1

26 ratio with adjuvants three times on day 0 , day 14 , and day 42 . All groups received the

27 vaccine intramuscularly (IM) with the exception of the c-di-AMP group that received

28 either the first vaccine IM and the second and third intranasally (IM-IN-IN) or all

29 vaccinations IN (IN-IN-IN). Previous work with other vaccines has shown these

30 regimens to be superior to IM-IM-IM protocols (Guzman; unpublished). Experiments

31 were done in accordance with the Canadian Council on Animal Care guidelines. 
1 Experimental methods were reviewed and approved by the University of Alberta Health

2 Sciences Animal Welfare Committee. Pre-vaccination serum was collected at day 0

3 before the first immunization and the final test bleed was collected two weeks after the

4 last immunization (Fig. 1). Serum was collected after centrifugation of the blood samples

5 at $5,000 \mathrm{xg}$ for 15 minutes. Serum was heat-inactivated by incubation at $56^{\circ} \mathrm{C}$ for 30

6 minutes and stored in aliquots at $-80^{\circ} \mathrm{C}$ until use.

rE2 ELISA: ELISA assays were performed as we described previously . Briefly,

8 96-well plates were coated with soluble rE2 (from amino acid \#384 to 656 of the H77

9 sequence) overnight at $4{ }^{\circ} \mathrm{C}$. Plates were washed and blocked for $1 \mathrm{~h}$ in $4 \%$ bovine serum

10 albumin (Sigma-Aldrich, St. Louis, MO, USA). Then, diluted antisera from vaccinated

11 mice were added to the plates for $1 \mathrm{~h}$. Finally, E2-specific antibodies from mouse antisera

12 were detected by a horseradish peroxidase-conjugated goat anti-mouse secondary

13 antibody (Cedarlane Laboratories, ON, Canada) and peroxidase substrate (KPL,

14 Gaithersburg, MD, USA). Absorbance values from three independent experiments are

15 expressed as means \pm standard errors of the means (SEM). Recombinant E2 H77 was

16 kindly provided by Joseph Marcotrigiano.

17 Neutralization assay: The ability of antibodies to neutralize HCV was evaluated 18 using HCV pseudo-particles (HCVpp) expressing genotype 1a (H77) E1E2 and carrying

19 a luciferase reporter gene. HCVpp was generated as described previously [35]. For

20 neutralization assay, human hepatoma cell line (Huh7.5) was seeded on collagen-coated

21 96-well plates 1 day prior to infection. The HCVpp stock was diluted 1:10, premixed

22 with either heat-inactivated $\left(56^{\circ} \mathrm{C}, 30 \mathrm{~min}\right)$ mouse sera or mouse anti-human $\mathrm{CD} 81$

23 monoclonal antibody (BD Biosciences, CA, USA) for $1 \mathrm{~h}$ at $37^{\circ} \mathrm{C}$, and then added to

24 Huh7.5 cell culture. The 96-well plates were spinoculated with a bench-top swing-bucket

25 centrifuge (Beckman Coulter, CA, USA) at $1,200 \mathrm{RPM}$ at $37^{\circ} \mathrm{C}$ for $1 \mathrm{~h}$, and then kept in a

$2637^{\circ} \mathrm{C}$ cell culture incubator (Thermo Fisher Scientific, MA, USA) for $6 \mathrm{~h}$. After

27 inoculation, media was replaced and the cells were incubated for additional $48 \mathrm{~h}$. Finally,

28 the cells were lysed and assessed for expression of luciferase using the Bright-Glo ${ }^{\mathrm{TM}}$

29 Luciferase Assay System (Promega, WI, USA) according to manufacturer's instructions.

30 Luminescence was measured with an EnSpire Multimode Plate Reader (PerkinElmer,

31 MA, USA). Neutralization activity was calculated as described previously [32]. 
T cell assays: After collecting the test bleeds, the spleens were immediately

2 extracted and collected in culture media. Splenocytes were immediately isolated and red

3 blood cells were lyzed using ACK RBC Lysis Buffer (Affymetrix eBiosciences, CA,

4 USA). The splenocytes from each group was pooled together (4 spleens per pool, 2 pools

5 per group) and dispensed in three replicates in the wells of a round bottom 96-well plate

6 (Corning, NY, USA) for negative control, positive control, and test groups. Each test

7 group was stimulated using a pool of 55 peptides spanning the whole homologous $\mathrm{H} 77$

8 HCV E1E2 region. Each peptide was 20 amino acid (aa) long overlapping 10 aa with up-

9 and down-stream peptides. The positive control groups received phorbol myristate

10 acetate (PMA) (250 ng/ml; Sigma, MO, USA) and Ionomycin ( $1 \mu \mathrm{g} / \mathrm{ml}$; Sigma) in

11 culture media and the negative control received media alone. DMSO was added at the

12 same volume as the test group $(0.4 \% \mathrm{v} / \mathrm{v})$ to the both negative and positive control

13 groups. Brefeldin A (Biolegend, CA, USA) and Monensin (Biolegend) were added to the

14 culture after 1 hour of incubation at $37^{\circ} \mathrm{C}$ and $5 \% \mathrm{CO} 2$. After additional $5 \mathrm{~h}$ of incubation

15 the cells were centrifuged and washed with PBS. The cells were then stained for

16 dead/live marker (Biolegend), surface markers (CD3, CD4, and CD8), intracellular

17 cytokines (IFN- $\gamma$ and TNF- $\alpha$ ) and finally were re-suspended in flow cytometry buffer

18 and read using Fortessa-SORP flow cytometer analyzer (BD Biosciences, CA, USA)

19 flow cytometer. The percentage of the T cells expressing both IFN- $\gamma$ and TNF- $\alpha$ were

20 compared between groups as an indicator of activated T cell responses to HCV rE1E2

21 antigen. The number of collected $\mathrm{CD}^{+} \mathrm{CD}^{+}$and $\mathrm{CD} 3^{+} \mathrm{CD} 8^{+}$cells were supportive of a

22 statistic $p$ value of 0.05 or less for each plot as described on Fig. 4 and Fig. 5.

23 Statistical Analysis: Data were analyzed using statistical software (GraphPad

24 Prism Version 5.00, Prism Software Corporation, Irvine, CA, USA). As outcome

25 variables from each group were not distributed normally, the medians between each pairs

26 of groups were compared using non-parametric Mann-Whitney test, where the ranks are

27 compared. Differences were considered significant if the two-tailed $P$ value was lower

28 than 0.05 with confidence intervals of $95 \%$.

30 Results 
Highly significant anti-E2 antibodies titers were detected by ELISA in all

2 vaccinated groups with exception of c-di-AMP (IN-IN-IN): As compared to the control

3 group (rE1E2 antigen alone), the sera collected from immunized mice with rE1E2 mixed

4 with different adjuvants showed significantly increased titers of anti-E1E2 ( $p$ value $<$

5 0.001) with the exception of the group rE1E2 + c-di-AMP (IN-IN-IN), where anti-E1E2

6 was not detected and the level of antibody was comparable to the group that only

7 received rE1E2 antigen with no adjuvant (Fig. 2). In the case of rE1E2 + c-di-AMP (IM-

8 IN-IN), the level of antibody was significantly lower than rE1E2 + MF59.

Antisera collected from immunized mice is able to neutralize and prevent the

11 the controls, the sera collected from immunized mice with adjuvanted H77 rE1E2

12 showed significant increases in preventing the entry of HCVpp in the in vitro

13 neutralization assay (Fig. 3)

14 When the antigen was formulated with Alum/MPLA or MF59 or c-di-AMP, this

15 effect was highly comparable to neutralization activity using a control anti-CD81

16 antibody that effectively blocks entry of HCVpp via the CD81 receptor. However,

17 although comparable to MF59 and Alum/MPLA, somewhat lower neutralizing antibodies

18 were measured in the case of c-di-AMP (IM-IN-IN) and archaeosomal vaccine

19 formulations when compared to anti-CD81 antibody. Comparison of c-di-AMP (IM-IN-

20 IN) and c-di-AMP (IN-IN-IN) groups showed that the route of immunization plays a

21 significant role, since mice vaccinated with three intranasal administrations (IN-IN-IN) of

22 rE1E2 + c-di-AMP did not neutralize HCVpp, in contrast to the regimen comprising an

23 intramuscular immunization followed by two intranasal boosts (IM-IN-IN). The relative

24 neutralizing activities of the different vaccine groups showed a similar trend to that

25 revealed in the ELISA assay.

Robust $\mathrm{CD4}^{+} \mathrm{T}$ cell immune responses were observed in c-di-AMP-and

27 archaeosomes-adjuvanted rE1E2 groups: In vitro stimulation of mice splenocytes with a

28 pool of 55 peptides that span the whole length of homologous HCV H77 E1E2 induced

29 strong activated $\mathrm{CD}^{+} \mathrm{T}$ cell responses in the groups where the antigen was formulated

30 with c-di-AMP or archaeosomes ( $p$ value $<0.005$ ). A moderate response was also

31 detected in the Alum/MPLA group ( $p$ value $<0.05$ ). Our data showed that c-di-AMP 
1 (IM/IN/IN) and archaeosomes elicit strong neutralizing antibodies comparable to MF59

2 and Alum/MPLA, and additionally both induced a more robust cellular immune response,

3 which was confirmed by the detection of vaccine specific poly-functional $\mathrm{CD} 4^{+} \mathrm{T}$ cells

4 expressing both IFN- $\gamma$ and TNF- $\alpha$ following in vitro stimulation (Fig. 4 and 5).

5 Interestingly, c-di-AMP elicited strong CD4+ T cell responses, even when given

6 IN/IN/IN unlike neutralizing antibody. None of the test groups induced a $\mathrm{CD} 8^{+} \mathrm{T}$ cellular

7 immune response (Fig.4 and 5).

\section{Discussion}

10 The data from this study indicates that modified H77 rE1E2 that is formulated

11 with Alum/MPLA, MF59, c-di-AMP, or archaeosomes is immunogenic in mice and can

12 induce the production of E1E2-specific antibodies with significant neutralization activity.

13 The presence of neutralizing activity is more indicative of protection against the pathogen

14 in comparison to ELISA antibody assays. Interestingly however, the data in both assays

15 correlated well. Strong neutralizing antibody activity is accompanied with very robust $\mathrm{T}$

16 cell immune responses when the antigen was formulated with c-di-AMP administered

$17 \mathrm{IM} / \mathrm{IN} / \mathrm{IN}$ or archaesomes given IM while a moderate $\mathrm{T}$ cell response was observed when

18 formulated with Alum/MPLA. This vaccine-specific cellular response was biased toward

$19 \mathrm{CD}^{+} \mathrm{T}$ cells and was not detected in the mice that were vaccinated with MF59-

20 adjuvanted $\mathrm{H} 77 \mathrm{rE} 1 \mathrm{E} 2$. This is highly compatible with our previous report, where the

21 sera collected from mice vaccinated with rE1E2-MF59 showed strong neutralizing

22 activity, but vaccination did not elicit any $\mathrm{CD}^{+}$or $\mathrm{CD}^{+} \mathrm{T}$ cellular immune response,

23 which was only detected when the $\mathrm{CpG}$ was added to the vaccine cocktail or where the

24 immunization protocol included at least one boost with replicon particles [36].

25 The absence of anti-E1E2 antibodies titers in ELISA and neutralizing assays (as

26 compared with antigen alone controls) in mice antisera in the rE1E2+c-di-AMP (IN-IN-

27 IN) group was surprising in view of previous data obtained with other antigens [37] but

28 this was a consistent finding in our hands.

29 With the exception of MF59, all other adjuvanted vaccines elicited

30 multifunctional CD4+ T cells producing both IFN $-\gamma$ and TNF- $\alpha$ production indicating

31 that the cellular response to $\mathrm{rE} 1 \mathrm{E} 2$ is accompanied with the generation of Th- 1 type CD4 ${ }^{+}$ 
1 T cells. In the sense of immune protection against $\mathrm{HCV}$, this is important as it is strongly

2 suggested that the presence of $\mathrm{HCV}$-specific Th-1 type $\mathrm{CD} 4^{+} \mathrm{T}$ cells correlates with the

3 spontaneous resolution of $\mathrm{HCV}$ infection, by facilitating the generation of $\mathrm{CD} 8^{+} \mathrm{T}$ cells

4 along with neutralizing antibodies [38-41]. Moreover, studies with Chimpanzee have

5 shown that the resolution of $\mathrm{HCV}$ during reinfection is highly dependent on $\mathrm{CD}^{+} \mathrm{T}$ cells

6 even thought the $\mathrm{CD} 8^{+} \mathrm{T}$ cells have been developed during the primary infection [42].

7 The detected $\mathrm{CD} 4^{+}$-specific cellular response in immunized mice may agree with our

8 previous finding in rE1E2-vaccinated humans [8], where we detected strong E1E2-

9 specific $\mathrm{CD}^{+} \mathrm{T}$ cell proliferation in peripheral blood mononuclear cells. The absence of

$10 \mathrm{a} \mathrm{CD}^{+}$-specific cellular response could be due to the absence of $\mathrm{CD}^{+} \mathrm{T}$ cell epitopes in

11 E1E2 in the inbred strain of mice used (CB6-F1) in comparison to human, where the

12 genetic diversity of MHC molecules in human may allow for additional $\mathrm{CD} 8^{+} \mathrm{T}$ cell

13 responses as suggested in a recent study [43].

14 Potent adjuvant activities of the di-nucleotide family (c-di-AMP and c-di-GMP)

15 have been shown before for the model antigen ovalbumin and recombinant antigens

16 derived from pathogens such as Streptococcus pneumoniae, Trypanosoma cruzii, or

17 influenza virus [25, 26, 44-47]. The use of the adjuvant c-di-AMP in a partial intranasal

18 immunization regimen has some advantages in avoiding repeated IM needle injections

19 for the recipient. However, no adjuvanted mucosal vaccines have been approved so far

20 partly due to prior safety concerns with other mucosal adjuvants [48-50]. The use of c-di-

21 AMP leads to the STING-dependent stimulation of both TNF-alpha and type I IFNs. In

22 general, the first cytokine seems to be critical for the stimulation of antibody responses

23 and the activation of Th2 cells [51], whereas type I IFNs are required for stimulation of

24 CTL responses and modulation of Th1 cells activity. Interestingly, c-di-AMP promotes

25 self-limited type I IFN activation as a result of an activation of STING degradation

26 (unpublished data). This feature, together with the capacity of c-di-AMP to promote a

27 type I IFN induction restricted at the local site of administration [52] reduces the

28 likelihood of systemic side effects related to immune activation thereby making c-di-

29 AMP a promising candidate adjuvant.

30 Archaeosome adjuvants comprised of total polar lipids of archaea target the

31 antigen effectively to APCs, and additionally provide co-stimulation and activation 
1 signals. The robust antigen-specific immune response including CD4+ T cells is

2 consistent with previous studies utilizing immunization of mice with model antigens

3 entrapped in various archaeosomes comprised of total polar lipids and/or a mixture of

4 semi-synthetic archaeal lipids. In this study, archaeosomes were comprised of a single

5 semi-synthetic negatively charged lipid (sulfated lactosyl archaeol), demonstrating the

6 advantage of a simple formulation. Archaeosomes traditionally also evoke robust CD8 T

7 cell response; however, the absence of the response may be related to lack of antigenic

8 epitopes in the mix. Moreover, archaeosomes in previous studies have been

9 demonstrated to evoke long-lasting functional responses to antigen, resulting in

10 prolonged efficacy against infectious or tumor challenge. Thus, comparing memory

11 immune responses to the various adjuvants may help select the appropriate formulation

12 for $\mathrm{HCV}$ vaccine.

13 In this study, we have presented a side-by-side comparison of the immunogenicity

14 of HCV rE1E2 formulated with c-di-AMP and archaeosomes versus two broadly used

15 and licensed adjuvants, MF59 and Alum/MPLA. We have demonstrated that in mice, c-

16 di-AMP and archaeosomes offer the advantage of inducing a superior cellular response.

17 This may indicate the use of c-di-AMP and archaeosomes as potential adjuvants to elicit

18 an optimal dual humoral and cellular immune response. In addition, our data also

19 indicates that use of a partial (but not exclusive) intranasal immunization regimen (IM-

20 IN-IN) may also be feasible using c-di-AMP as adjuvant.

\section{Acknowledgments}

23 This work was funded by the Li Ka Shing Applied Virology Institute at the

24 University of Alberta with grants from Alberta Innovates Health Solutions.

\section{References} HCV infection. Nat Rev Gastroenterol Hepatol, 2013. 10(9): p. 553-62.

2. Chung, R.T. and T.F. Baumert, Curing chronic hepatitis $C$--the arc of a medical triumph. N Engl J Med, 2014. 370(17): p. 1576-8.

3. Callaway, E., Hepatitis $C$ drugs not reaching poor. Nature, 2014. 508(7496): p. 295-6. 
14 Gozlan, Y., et al., [Resistance of Hcv to New Direct Acting Antivirals]. Harefuah, 2 2015. 154(11): p. 684-7, 743.

3 5. Midgard, H., et al., Hepatitis C reinfection after sustained virological response. J

6. Cox, A.L., MEDICINE. Global control of hepatitis C virus. Science, 2015. 349(6250): p. 790-1.

7. Shahzad, A. and G. Kohler, Inactivated Polio Vaccine (IPV): a strong candidate vaccine for achieving global polio eradication program. Vaccine, 2009. 27(39): p. 5293-4.

8. Frey, S.E., et al., Safety and immunogenicity of HCV E1E2 vaccine adjuvanted with MF59 administered to healthy adults. Vaccine, 2010. 28(38): p. 6367-73.

9. Houghton, M., Prospects for prophylactic and therapeutic vaccines against the hepatitis $C$ viruses. Immunological reviews, 2011. 239(1): p. 99-108.

10. Ray, R., et al., Characterization of antibodies induced by vaccination with hepatitis $C$ virus envelope glycoproteins. The Journal of infectious diseases, 2010. 202(6): p. 862-6.

11. Law, J.L., et al., $A$ hepatitis $C$ virus (HCV) vaccine comprising envelope glycoproteins gpE1/gpE2 derived from a single isolate elicits broad crossgenotype neutralizing antibodies in humans. PLoS One, 2013. 8(3): p. e59776.

12. Goto, N., et al., Local tissue irritating effects and adjuvant activities of calcium phosphate and aluminium hydroxide with different physical properties. Vaccine, 1997. 15(12-13): p. 1364-71.

13. Morefield, G.L., et al., Role of aluminum-containing adjuvants in antigen internalization by dendritic cells in vitro. Vaccine, 2005. 23(13): p. 1588-95.

14. Kool, M., et al., Cutting edge: alum adjuvant stimulates inflammatory dendritic cells through activation of the NALP3 inflammasome. J Immunol, 2008. 181(6): p. 3755-9.

15. Kool, M., et al., Alum adjuvant boosts adaptive immunity by inducing uric acid and activating inflammatory dendritic cells. J Exp Med, 2008. 205(4): p. 86982.

16. Agger, E.M., et al., Adjuvant modulation of the cytokine balance in Mycobacterium tuberculosis subunit vaccines; immunity, pathology and protection. Immunology, 2008. 124(2): p. 175-85.

17. Garcon, N., et al., Development of an ASO4-adjuvanted HPV vaccine with the adjuvant system approach. BioDrugs, 2011. 25(4): p. 217-26.

18. Harper, D.M., Currently approved prophylactic HPV vaccines. Expert Rev Vaccines, 2009. 8(12): p. 1663-79.

19. Galli, G., et al., Adjuvanted H5N1 vaccine induces early CD4+ T cell response that predicts long-term persistence of protective antibody levels. Proc Natl Acad Sci U S A, 2009. 106(10): p. 3877-82.

20. Mosca, F., et al., Molecular and cellular signatures of human vaccine adjuvants. Proc Natl Acad Sci U S A, 2008. 105(30): p. 10501-6.

21. Dupuis, M., et al., Dendritic cells internalize vaccine adjuvant after intramuscular injection. Cell Immunol, 1998. 186(1): p. 18-27.

22. Burdette, D.L., et al., STING is a direct innate immune sensor of cyclic di-GMP. Nature, 2011. 478(7370): p. 515-8. 
23. Woodward, J.J., A.T. Iavarone, and D.A. Portnoy, c-di-AMP secreted by intracellular Listeria monocytogenes activates a host type I interferon response. Science, 2010. 328(5986): p. 1703-5.

24. Karaolis, D.K., et al., 3',5'-Cyclic diguanylic acid (c-di-GMP) inhibits basal and growth factor-stimulated human colon cancer cell proliferation. Biochem Biophys Res Commun, 2005. 329(1): p. 40-5.

25. Ebensen, T., et al., The bacterial second messenger cyclic diGMP exhibits potent adjuvant properties. Vaccine, 2007. 25(8): p. 1464-9.

26. Ebensen, T., et al., Bis-(3',5')-cyclic dimeric adenosine monophosphate: strong Th1/Th2/Th17 promoting mucosal adjuvant. Vaccine, 2011. 29(32): p. 521020.

27. Haq, K., Y. Jia, and L. Krishnan, Archaeal lipid vaccine adjuvants for induction of cell-mediated immunity. Expert Rev Vaccines, 2016. 15(12): p. 1557-1566.

28. Patel, G.B., et al., In vitro assessment of archaeosome stability for developing oral delivery systems. Int J Pharm, 2000. 194(1): p. 39-49.

29. Patel, G.B. and G.D. Sprott, Archaeobacterial ether lipid liposomes (archaeosomes) as novel vaccine and drug delivery systems. Crit Rev Biotechnol, 1999. 19(4): p. 317-57.

30. Krishnan, L., et al., Archaeosome vaccine adjuvants induce strong humoral, cell-mediated, and memory responses: comparison to conventional liposomes and alum. Infect Immun, 2000. 68(1): p. 54-63.

31. Krishnan, L. and G.D. Sprott, Archaeosome adjuvants: immunological capabilities and mechanism(s) of action. Vaccine, 2008. 26(17): p. 2043-55.

32. Logan, M., et al., Native Folding of a Recombinant gpE1/gpE2 Heterodimer Vaccine Antigen from a Precursor Protein Fused with Fc IgG. J Virol, 2017. 91(1).

33. Rigaud, J.L., et al., Detergent removal by non-polar polystyrene beads. European Biophysics Journal, 1998. 27(4): p. 305-319.

34. Schubert, R., Liposome preparation by detergent removal. Methods Enzymol, 2003. 367: p. 46-70.

35. Hsu, M., et al., Hepatitis $C$ virus glycoproteins mediate $p H$-dependent cell entry of pseudotyped retroviral particles. Proc Natl Acad Sci U S A, 2003. 100(12): p. 7271-6.

36. Lin, Y., et al., Induction of broad CD4+ and CD8+ T-cell responses and crossneutralizing antibodies against hepatitis $C$ virus by vaccination with Th1adjuvanted polypeptides followed by defective alphaviral particles expressing envelope glycoproteins gpE1 and gpE2 and nonstructural proteins 3, 4, and 5. Journal of virology, 2008. 82(15): p. 7492-503.

37. Akbar, S.M., et al., Dendritic cells and chronic hepatitis virus carriers. Intervirology, 2001. 44(4): p. 199-208.

38. Della Bella, S., et al., Decrease and dysfunction of dendritic cells correlate with impaired hepatitis $C$ virus-specific CD4+ T-cell proliferation in patients with hepatitis $C$ virus infection. Immunology, 2007. 121(2): p. 283-92.

39. Miyatake, H., et al., Impaired ability of interferon-alpha-primed dendritic cells to stimulate Th1-type CD4 T-cell response in chronic hepatitis $C$ virus infection. J Viral Hepat, 2007. 14(6): p. 404-12. 
40. Waggoner, S.N., C.H. Hall, and Y.S. Hahn, HCV core protein interaction with gC1q receptor inhibits Th1 differentiation of CD4+ T cells via suppression of dendritic cell IL-12 production. J Leukoc Biol, 2007. 82(6): p. 1407-19.

41. Chang, K.M., et al., Differential CD4(+) and CD8(+) T-cell responsiveness in hepatitis $C$ virus infection. Hepatology, 2001. 33(1): p. 267-76.

42. Grakoui, A., et al., HCV persistence and immune evasion in the absence of memory T cell help. Science, 2003. 302(5645): p. 659-62.

43. Filskov, J., et al., Broadening CD4+ and CD8+ T cell responses against hepatitis $C$ virus by vaccination with NS3 overlapping peptide panels in cross-priming liposomes. J Virol, 2017.

44. Ogunniyi, A.D., et al., $c$-di-GMP is an effective immunomodulator and vaccine adjuvant against pneumococcal infection. Vaccine, 2008. 26(36): p. 4676-85.

45. Matos, M.N., et al., Immunization with Tc52 or its amino terminal domain adjuvanted with c-di-AMP induces Th17+Th1 specific immune responses and confers protection against Trypanosoma cruzi. PLoS Negl Trop Dis, 2017. 11(2): p. e0005300.

46. Skrnjug, I., C.A. Guzman, and C. Rueckert, Cyclic GMP-AMP displays mucosal adjuvant activity in mice. PLoS One, 2014. 9(10): p. e110150.

47. Sanchez, M.V., et al., Intranasal delivery of influenza rNP adjuvanted with c-diAMP induces strong humoral and cellular immune responses and provides protection against virus challenge. PLoS One, 2014. 9(8): p. e104824.

48. Mutsch, M., et al., Use of the inactivated intranasal influenza vaccine and the risk of Bell's palsy in Switzerland. N Engl J Med, 2004. 350(9): p. 896-903.

49. Fujihashi, K., et al., A dilemma for mucosal vaccination: efficacy versus toxicity using enterotoxin-based adjuvants. Vaccine, 2002. 20(19-20): p. 2431-8.

50. Glueck, R., Pre-clinical and clinical investigation of the safety of a novel adjuvant for intranasal immunization. Vaccine, 2001. 20 Suppl 1: p. S42-4.

51. Blaauboer, S.M., V.D. Gabrielle, and L. Jin, MPYS/STING-mediated TNF-alpha, not type I IFN, is essential for the mucosal adjuvant activity of ( $3^{\prime}-5$ ')-cyclic-diguanosine-monophosphate in vivo. J Immunol, 2014. 192(1): p. 492-502.

52. Rueckert, $\mathrm{C}$., et al., Cyclic dinucleotides modulate induced type I IFN responses in innate immune cells by degradation of STING. FASEB J, 2017.

Figure Legends

36

Figure 1. The experimental plan

39 Figure 2. Detection of anti-HCV E2 in mice immunized with rE1E2. Highly significant 40 anti-E2 antibodies titers were detected by ELISA in all vaccinated groups with exception

41 of c-di-AMP (IN-IN-IN). Horizontal lines are the medians in each group. ${ }^{* * *}=p$

42 value $<0.001 ;^{* *}=p$ value $<0.01 ;$ and $^{*}=p$ value $<0.05$. 
2 Figure 3. Neutralization activity of rE1E2 in combination with adjuvant in mice. The

3 sera from mice immunized with rE1E2 are able to neutralize the entry of HCV

4 pseudo particle in vitro. Percentage of neutralization was calculated based on the

5 neutralizing activity for post-vaccination bleed divided by neutralization activity of

6 pre-vaccination bleed. Horizontal lines are the medians in each group. ${ }^{* *}=p$ value $<$

$7 \quad 0.01 ;$ and ${ }^{*}=p$ value $<0.05$.

9 Figure 4. Differential detection of T cells immune response in mice. The splenocytes

10 from vaccinated mice with rE1E2 in combination with different adjuvants were

11 stimulated in vitro and intracellular production of cytokine was detected by multi-

12 color flow cytometry. IM = Intramuscular; IN = Intranasal; Control = Negative

13 control splenocytes; Peptide pool = Splenocytes that are stimulated with a pool of 55

14 peptides spanning E1E2.

16 Figure 5. The percentage of $\mathrm{CD}^{+}$and $\mathrm{CD}^{+} \mathrm{T}$ cells that are expressing INF- $\gamma$, TNF- $\alpha$

17 or both. Graphs $\mathbf{a}, \mathbf{b}$, and $\mathbf{c}$ showing CD $4^{+}$T cells and graphs $\mathbf{d}, \mathbf{e}$, and $\mathbf{f}$ showing CD ${ }^{+}$

18 T cells. The splenocytes from vaccinated mice with rE1E2 in combination with

19 different adjuvants were stimulated in vitro and intracellular production of cytokine

20 was detected by multi-color flow cytometry. IM = Intramuscular; IN = Intranasal;

21 Control $=$ Negative control splenocytes; Peptide pool $=$ Splenocytes that are

22 stimulated with a pool of 55 peptides spanning E1E2. 


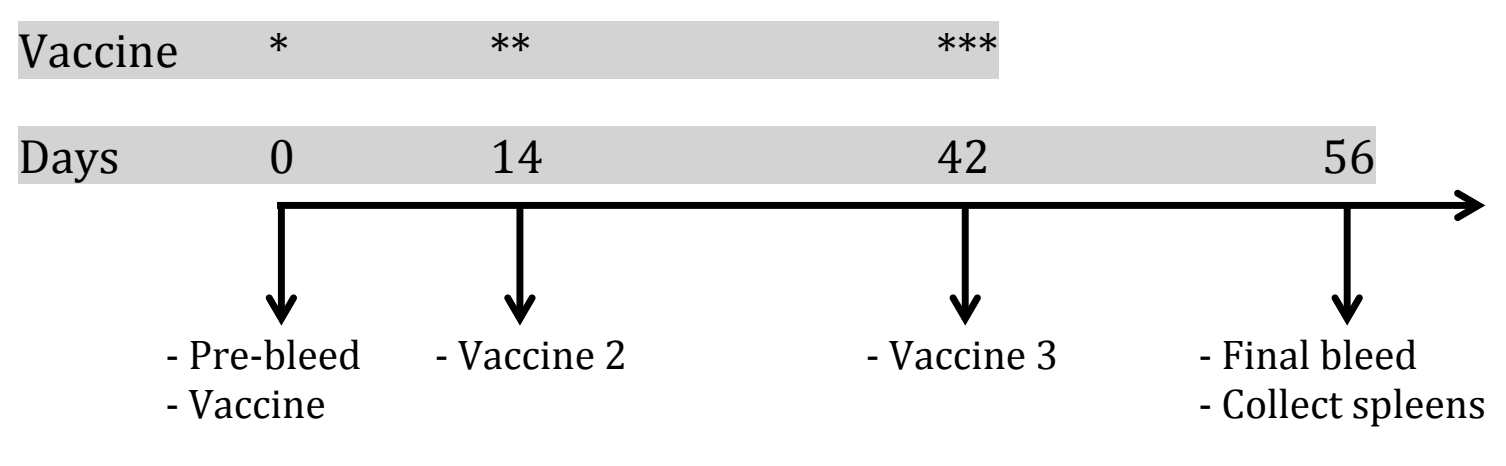

Figure 1. Landi et al.

- Vaccine

- Collect spleens 
rE2 (384-656) H77 ELISA

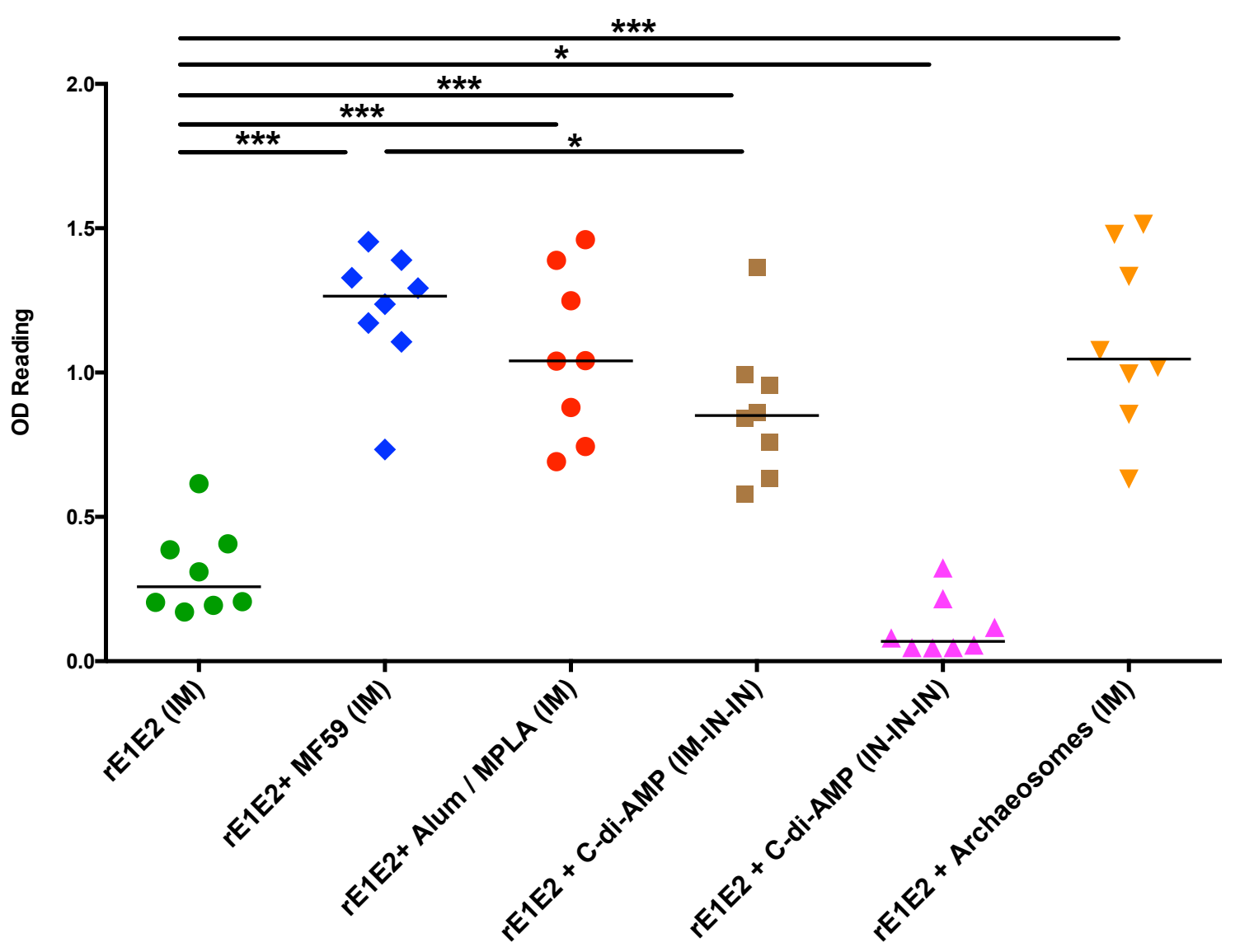

Figure 2. Landi et al. 
HCVpp Neutralization H77

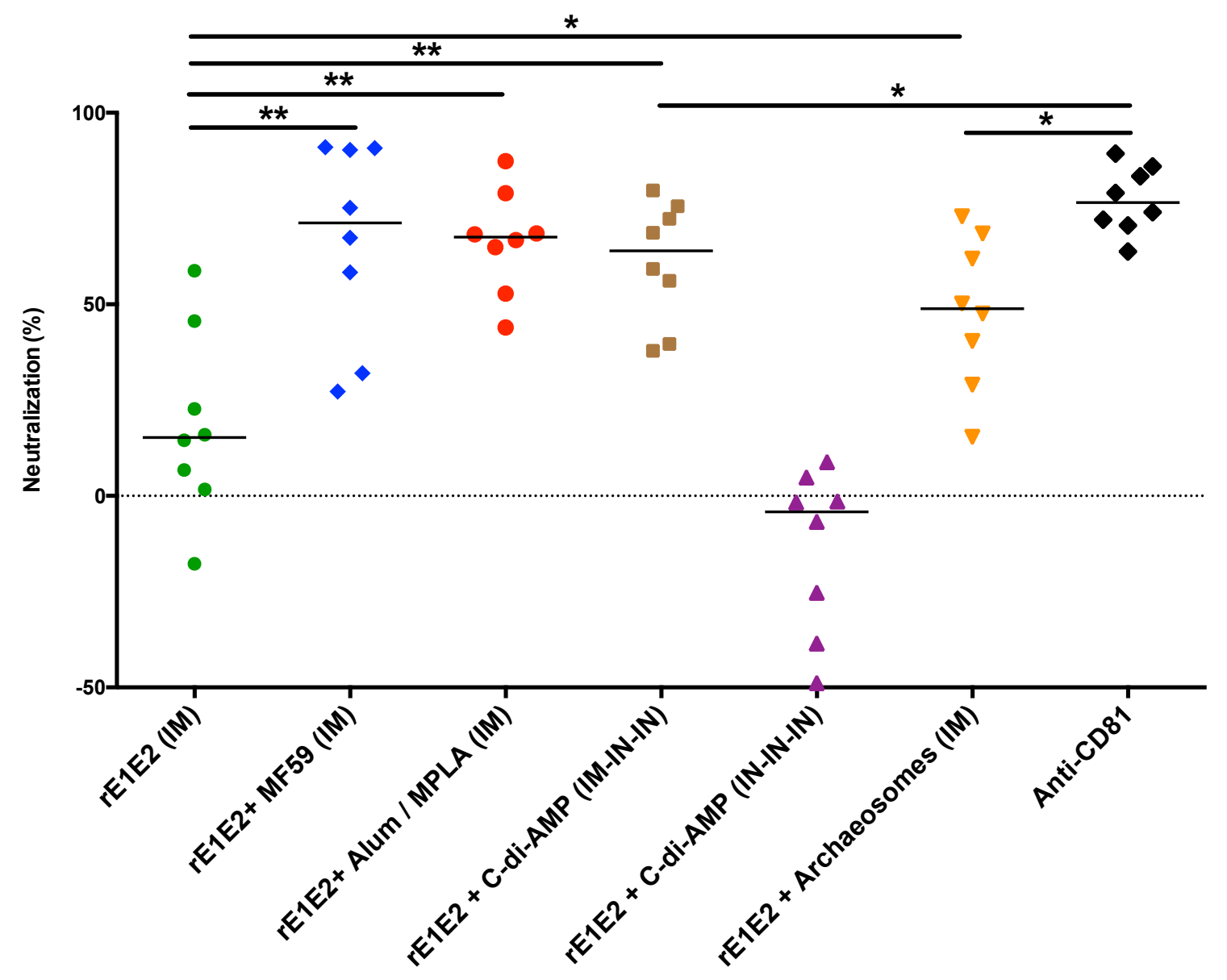

Figure 3. Landi et al. 


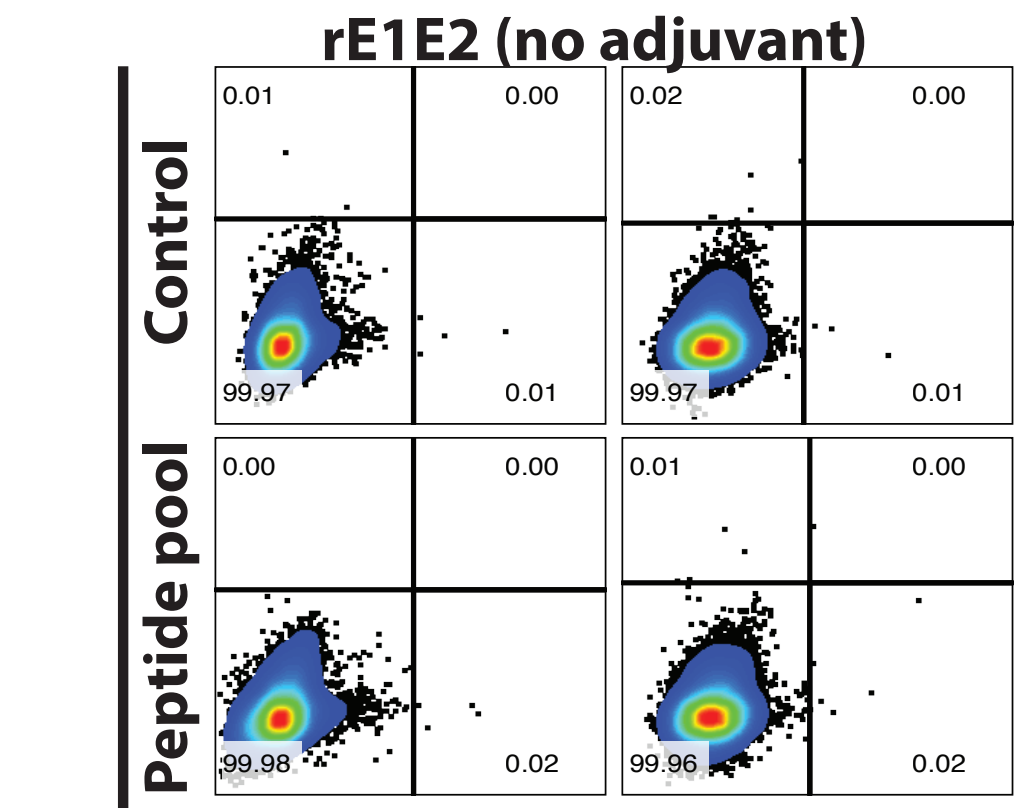

\begin{tabular}{c} 
rE1E2 + c-di-AMP (IM-IN-IN) \\
\hline 0.01
\end{tabular}

rE1E2 + MF59

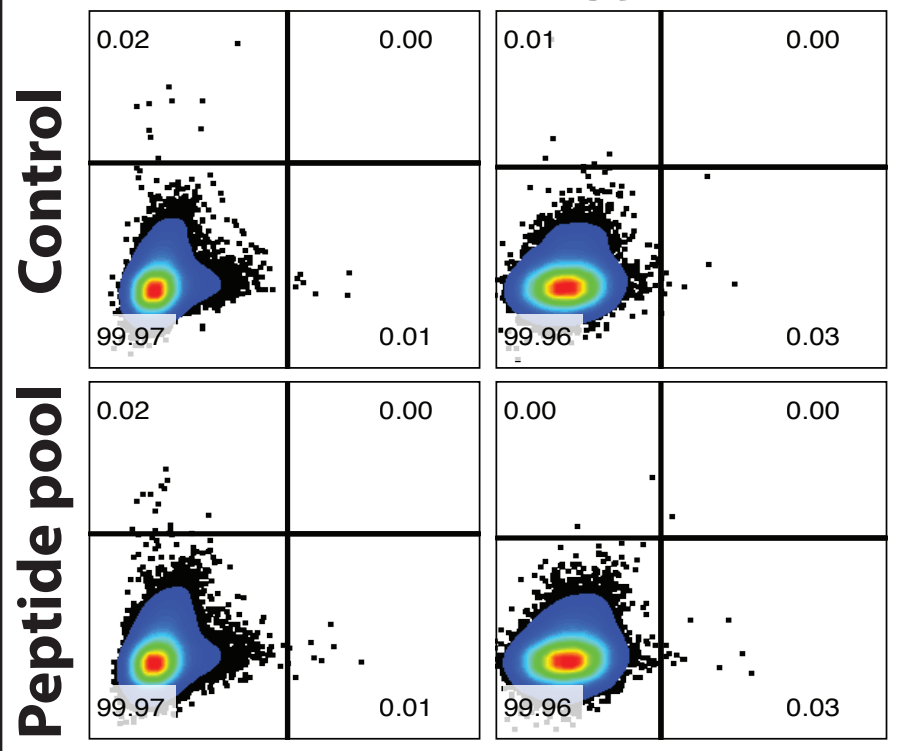

rE1E2 + Alum/MPLA

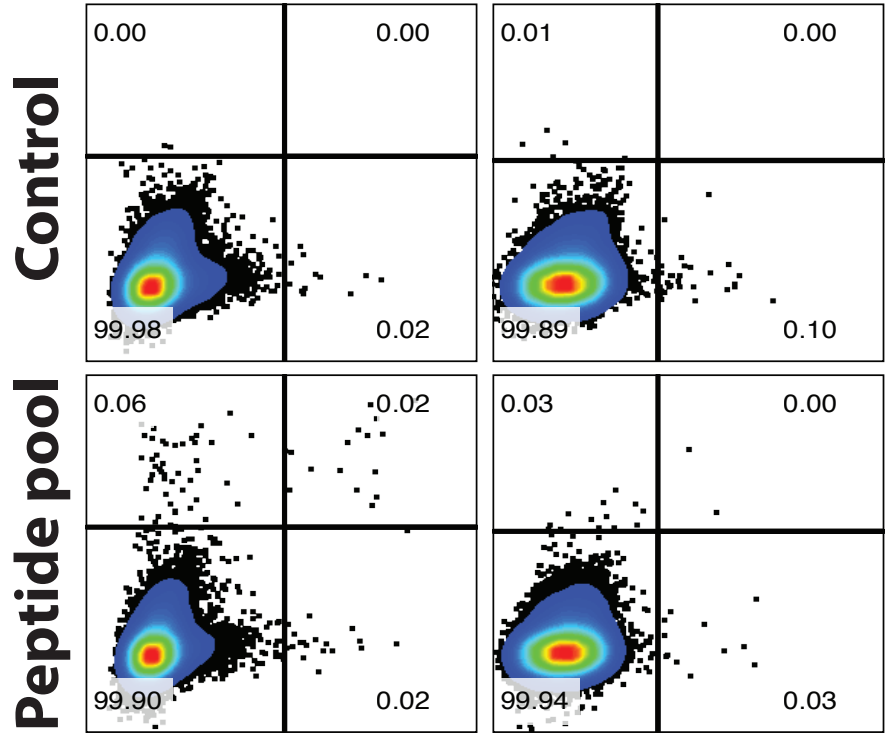

rE1E2 + c-di-AMP (IN-IN-IN)

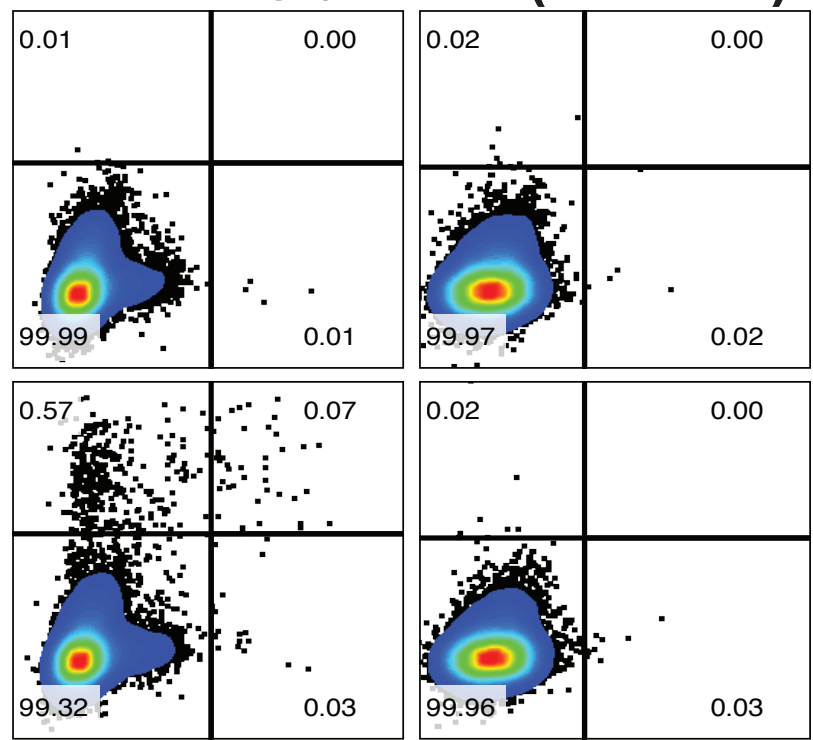

rE1E2 + archaesomes

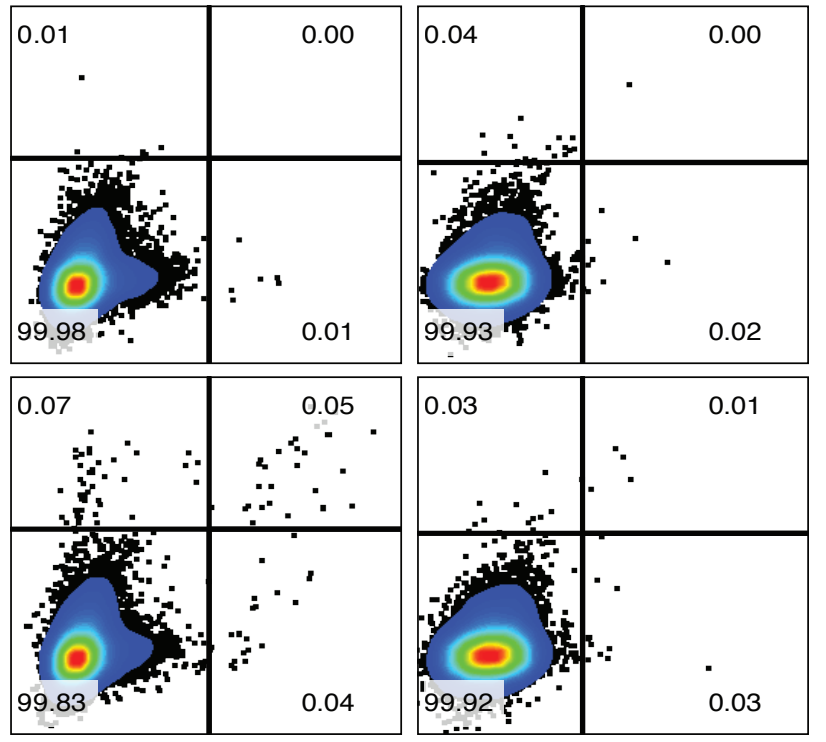



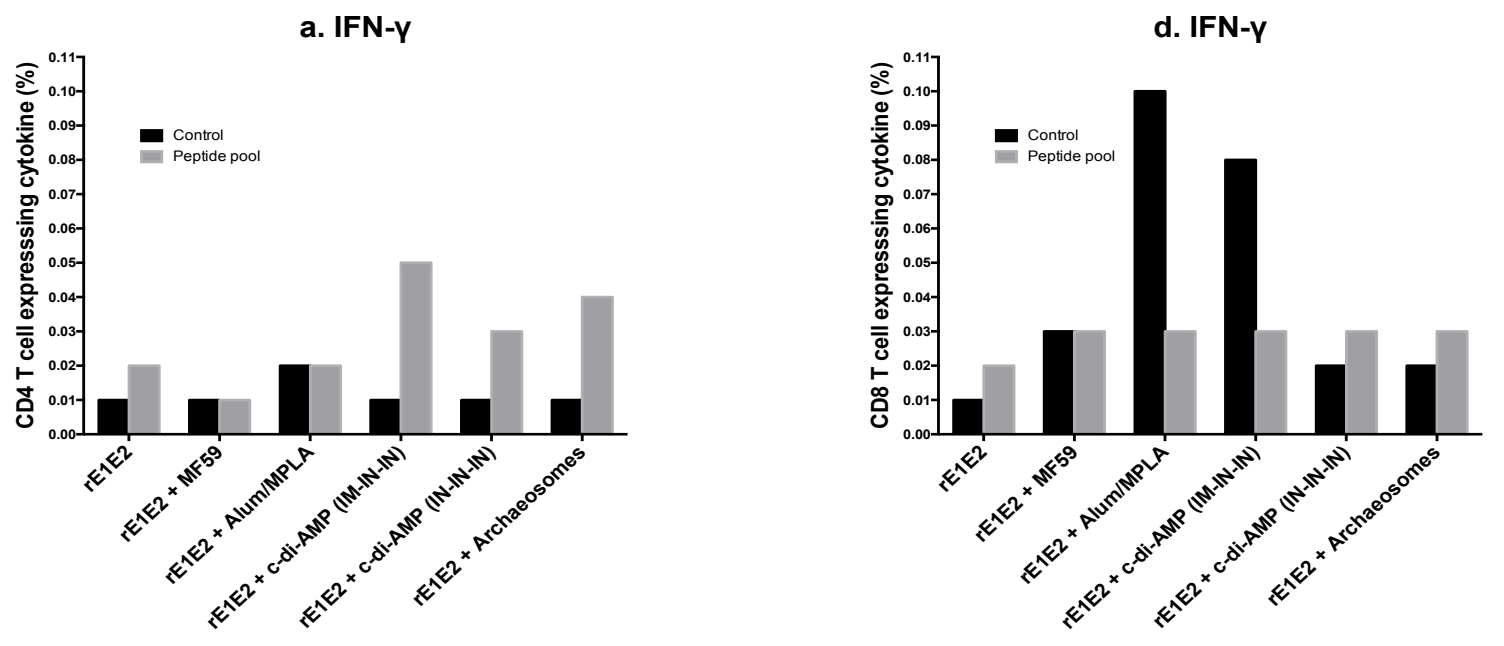

b. TNF- $\alpha$

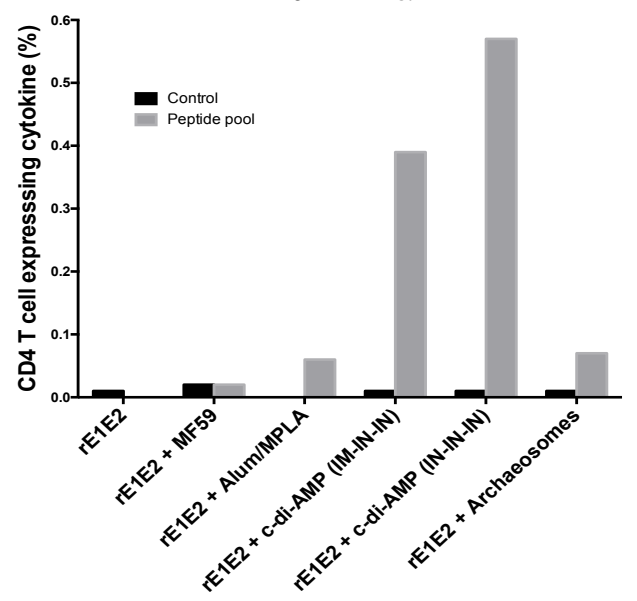

c. IFN-y + TNF- $\alpha$

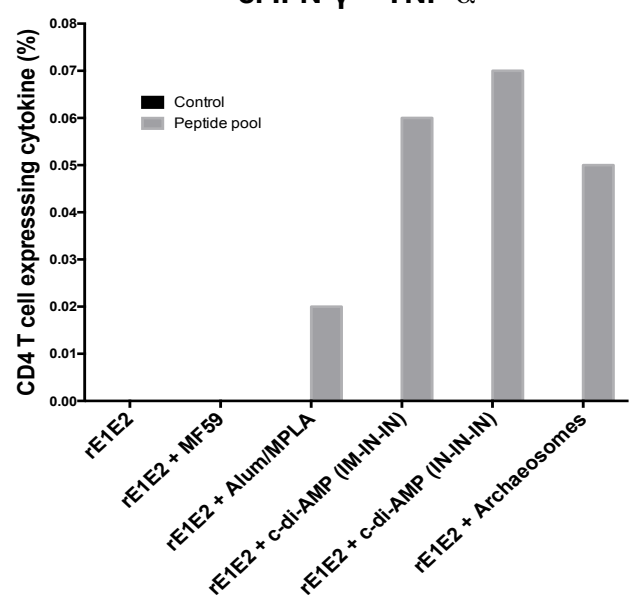

e. TNF- $\alpha$

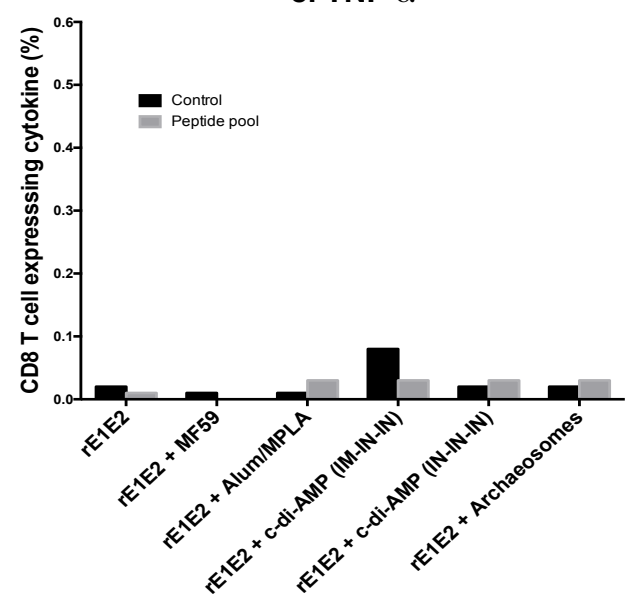

f. IFN- $y+$ TNF- $\alpha$

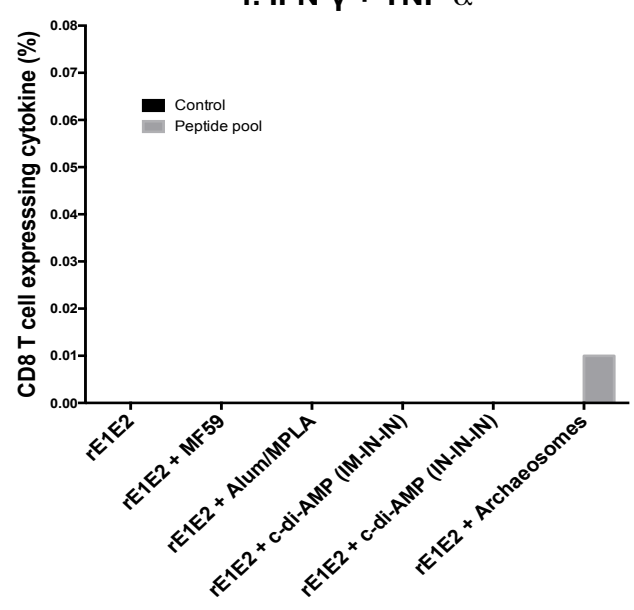

Figure 5. Landi et al. 
